\title{
lieuxdits \#17
}

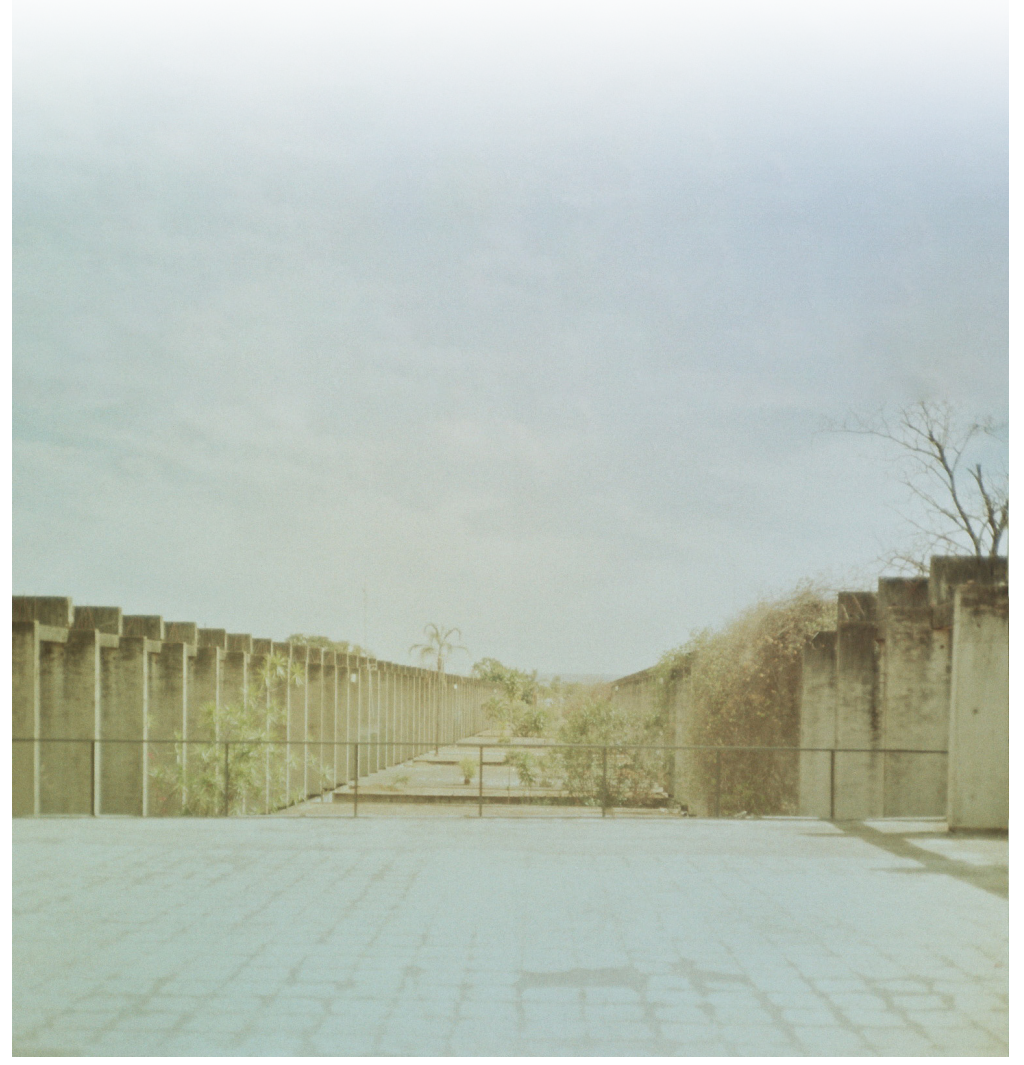

Référence bibliographique :

Fabrice Noukpakou, Renaud Pleitinx, "Le paysage Otammari", lieuxdit\$17, décembre 2019, pp. 21-24.

\section{La revue lieuxdits}

Faculté d'architecture, d'ingénierie architecturale, d'urbanisme (LOCI)

Univesrité catholique de Louvain (UCL).

Éditeur responsable : Le comité de rédaction, place du Levant, 1 - 1348 Louvain-la-Neuve

Comité de rédaction : Damien Claeys, Gauthier Coton, Pauline Fockedey,

Nicolas Lorent, Guillaume Vanneste

Conception graphique : Nicolas Lorent

\section{(1)}




\title{
Le paysage Otammari
}

\author{
Fabrice Noukpakou, Renaud Pleitinx
}

La recherche doctorale que nous conduisons en Loci, sous la direction des professeurs Renaud Pleitinx et Quentin Wilbaux, est née de la rencontre de notre propre intérêt pour le patrimoine architectural du Bénin et, en particulier, l'habitat otammari, avec les exigences et les compétences des cellules de recherche auxquelles appartiennent nos deux promoteurs : le laboratoire analyse architecture (laa) et LoCiLoCaL.

Le laa coordonné par les professeurs Cécile Chanvillard, Pierre Cloquette, Renaud Pleitinx et Jean Stillemans est spécialisé dans le domaine de la théorie de l'architecture. Il se donne pour tâche de définir et de comprendre l'architecture en vue de fournir aux chercheurs dans les domaines de l'architecture des critères d'observation et des principes d'explication utiles à l'étude de toute espèce d' habitat.

Coordonné, par le professeur Quentin Wilbaux, le LoCiLoCaL conduit des recherches dans les domaines de l'architecture, de l'urbanisme et de l'aménagement du territoire avec comme souci de tirer le meilleur parti des ressources locales. Dans le cadre de ses recherches, le LoCiLoCal met au point et met en cuvre des techniques de relevé qui font appel à la photographie aérienne (par avion ou par drone) et à la photogrammétrie.

Notre recherche exploite, d'une part, les outils d'analyse et les schèmes explicatifs proposés par le laa et met en ceuvre, d'autre part, les techniques de relevé développées et utilisées au sein de LoCiLoCaL. Pour présenter l'état de cette recherche en cours, nous en préciserons successivement l'objet, la problématique et la méthode.

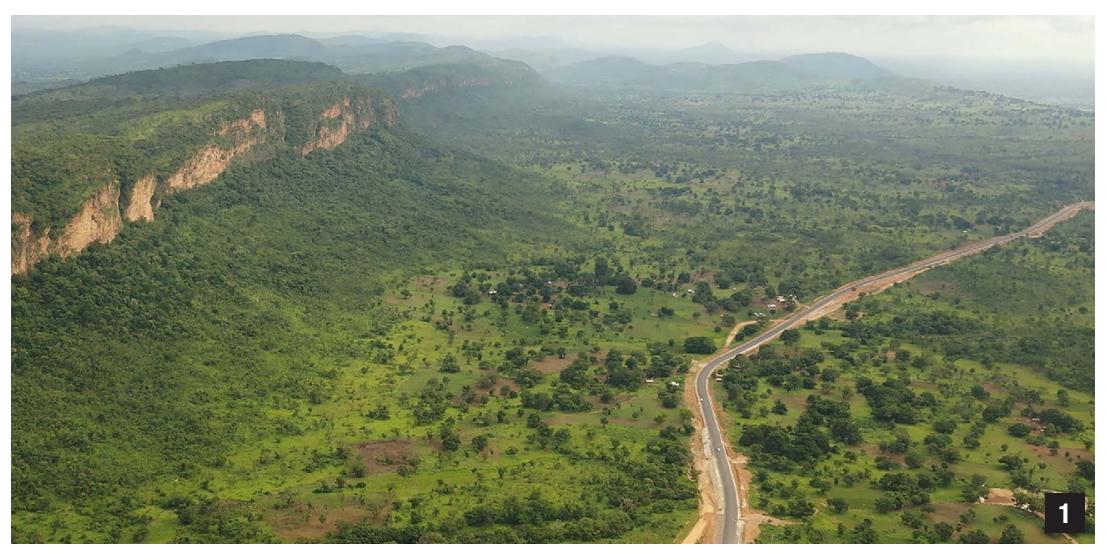

\section{Objet}

L'objet de notre recherche est l'habitat otammari. Le peuple otammari vit dans la région montagneuse et rurale de l'Atacora (fig. 1) située au nord-ouest du Bénin. Le terme otammari (pl. : Bètammaribè) signifie "architecte" ou "bon maçon"1, il s'applique aussi bien à la personne, l'otammari, chargée d'élaborer et d'exécuter les ouvrages qu'au peuple otammari tout entier. Les Bètammaribè composent avec les Bètiabè, et les Bèsorbè (Bénin), ainsi que les Tamberma (Togo) l'ethnie somba. Les recherches ethnohistoriques de Paul Mercier² et archéologiques de Didier N'Dah ${ }^{3}$ retracent I'histoire de leurs migrations depuis le Burkina Faso. Venus d'horizons divers par vagues successives entre le $\mathrm{Xvl}^{\mathrm{e}}$ et le $\mathrm{XVIII}$ e siècle, se sont installés dans l'Atacora de petits groupes qui ont conservé les usages des sociétés qu'ils ont côtoyées, et ont formé un peuple hétérogène fortement ancré dans ses pratiques cérémonielles.

La takienta (fig. 2) est devenue l'emblème de l'habitat otammari. Usuellement, la takienta est composée, de plusieurs cases circulaires, construites en terre crue, (d'approximativement deux mètres de diamètre) coiffées de toitures coniques recouvertes de paille. Disposées en cercle, ces cases sont reliées entre elles par des murs courbes construits, eux aussi, en terre crue. L'ensemble mesure de trois à quatre mètres de haut, car la particularité de la takienta est de posséder un étage. Ce dernier est matérialisé par une, voire plusieurs, terrasse en terre damée, supportée par des poteaux et des poutres en bois. La terrasse principale donne accès aux chambres et aux greniers implantés à l'étage. Avec ses cases en forme de tourelles, avec ses hauts murs de défense, la silhouette de la takienta évoque, aux yeux de certains, celle des châteaux forts ${ }^{4}$.
1 L'Atacora (photographie: Yves Baudot, Fabrice Noukpakou)

1 - PUREN, Odile, « Le tata somba Châteaux forfifié du nord-ouest du Bénin », dans La revue de Téhéran, $\mathrm{n}$ 53,2010, pp. 1-12.

2 - MERCIER, Paul, Tradition, changement, histoire: les "Somba" du Dahomey septentrional, Paris, Ed. Anthropos, 1968

3 - N'DAH, Didier, Sites archéo logiques et peuplement de la région de l'Atakora (nord-ouest du Bénin) Ouagadougou, Université de Ouagadougou, Département d'histoire et archéologie, 2009

4-MERCIER, Paul, L'habitation (áétages) dans l'Atakora, Cotonou, Office de la recherche scientifique et technique outre-mer, 1954. 
Takienta typique (photogrammétrie: Yves Baudot, Fabrice Noukpakou

3 Schéma génératif simplifié de l’organisation des Takientè (dessin : Fabrice Noukpakou, Renaud Pleitinx)

\section{5 - MERCIER, Paul (1954), op.cit}

6- BLIER, Suzan Preston, The Anatomy of Architecture: Ontology and Metaphor in Batammaliba Architectural Expression, Chicago, University of Chicago Press, 1994 (édition originale 1987), coll. « Res monographs in anthropology and aesthetics ». PADENOU, Guy-Herman et Monique PASTOR-BARRUÉ, Architecture, société et paysage Bétammaribé au Togo: contribution a l'anthropologie del'habitat, Toulouse, Presses universitaires du Mirail, 2006

7 - GAGNEPAIN, Jean, Du vouloir dire: Traité d'épistémologie des sciences bumaines: I: Dusigne, de l'outil, Paris, Livre \& Communication, 1990 (édition originale 1982), coll. «Épistémologie ».

PJONGEN, René, Quand dire cest dire: Initiation à une linguistique glossologique et a l'anthropologie clinique. Bruxelles, De Boeck Université, 1993, coll. « Raisonnances».

PLEITINX, Renaud, « Des raisons du lieu », dans Laboratoire Analyse Architecture, 2006, pp. 1-41.

8 - PLEITINX, Renaud et Sèdjro Houègbonou NOUKPAKOU, L'adaptabilité de l'architecture otàmmari, Cotonou, 2019, coll. «Vivre en Ville - Vivre la Ville».

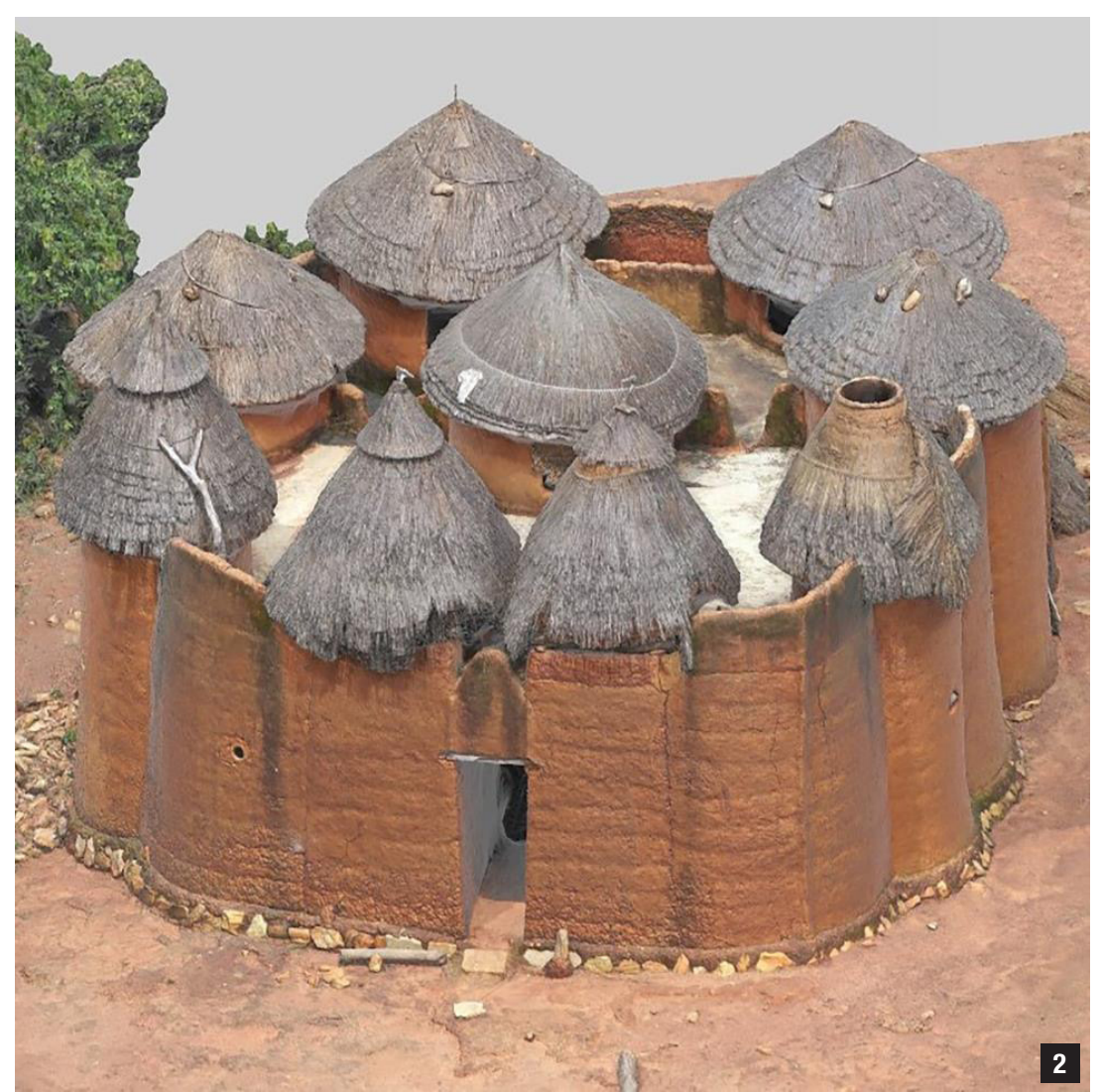

\section{Problématique}

La takienta est décrite dans la littérature spécialisée comme une unité d'habitation unifamiliale autonome. Tous les membres de la famille y trouveraient leur logement : le rez-de-chaussée serait destiné aux animaux domestiques et aux personnes âgées, tandis que les pièces et les greniers à l'étage seraient répartis entre le père de famille, ses épouses et leurs enfants.

Considérant la takienta comme un ouvrage isolé ou focalisant leurs analyses sur l'ouvrage le plus emblématique de l'architecture otammari, la plupart des auteurs privilégient un traitement taxinomique (typologique) de leur objet. Ils relèvent ainsi les traits caractéristiques des ouvrages (courbure des murs, hauteurs des tourelles, inclinaison de la terrasse, etc.) et, plus loin, répertorient leurs variations, qui sont ensuite attribuées à l'un ou l'autre des groupes socioculturels qui composent l'ethnie somba ${ }^{5}$. Ce faisant, ils en viennent à définir des archétypes ou des "modèles" 6 auxquels sont censés correspondre les ouvrages attestés.

Plutôt qu'une approche taxinomique, nous avons adopté une approche générative ${ }^{7}$. Au lieu d'étudier les traits caractéristiques et les variations d'un modèle, nous nous sommes proposé d'étudier les unités et les principes d'articulation de I'habitat otammari (fig. 3). Dès lors, il ne s'agit pas, pour nous, de considérer la takienta comme une entité intègre et séparée de toute autre, mais bien

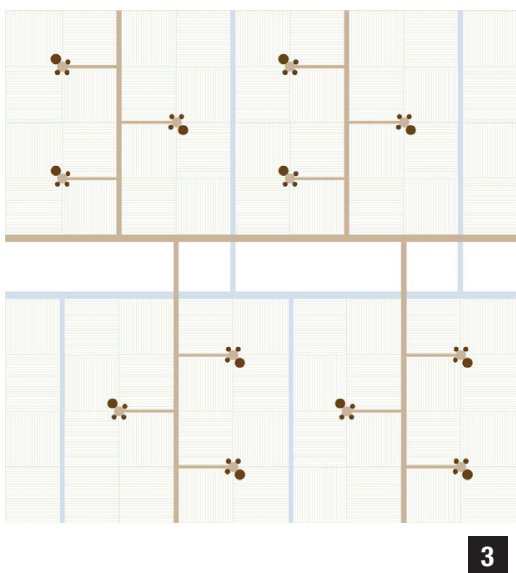

comme une entité constituée en ellemême d'unités élémentaires, et comme une entité constitutive au-delà d'ellemême d'ensembles plus étendus ${ }^{8}$.

Ces ensembles sont, en l'occurrence : la concession; le domaine; le village; le territoire. La concession, dont les dimensions se mesurent en mètres, comprend, outre la takienta, la cour qui est l'espace de vie principal des Bètammaribè. C'est dans cette cour que se trouvent le foyer principal et les autels, et c'est autour de cette cour que se distribuent la paillote, les éventuelles cases supplémentaires, les fours à karité, etc. Le domaine, qui se mesure en dizaines de mètres, comprend, outre la concession, les terres agricoles qui la bordent. De ces terres 


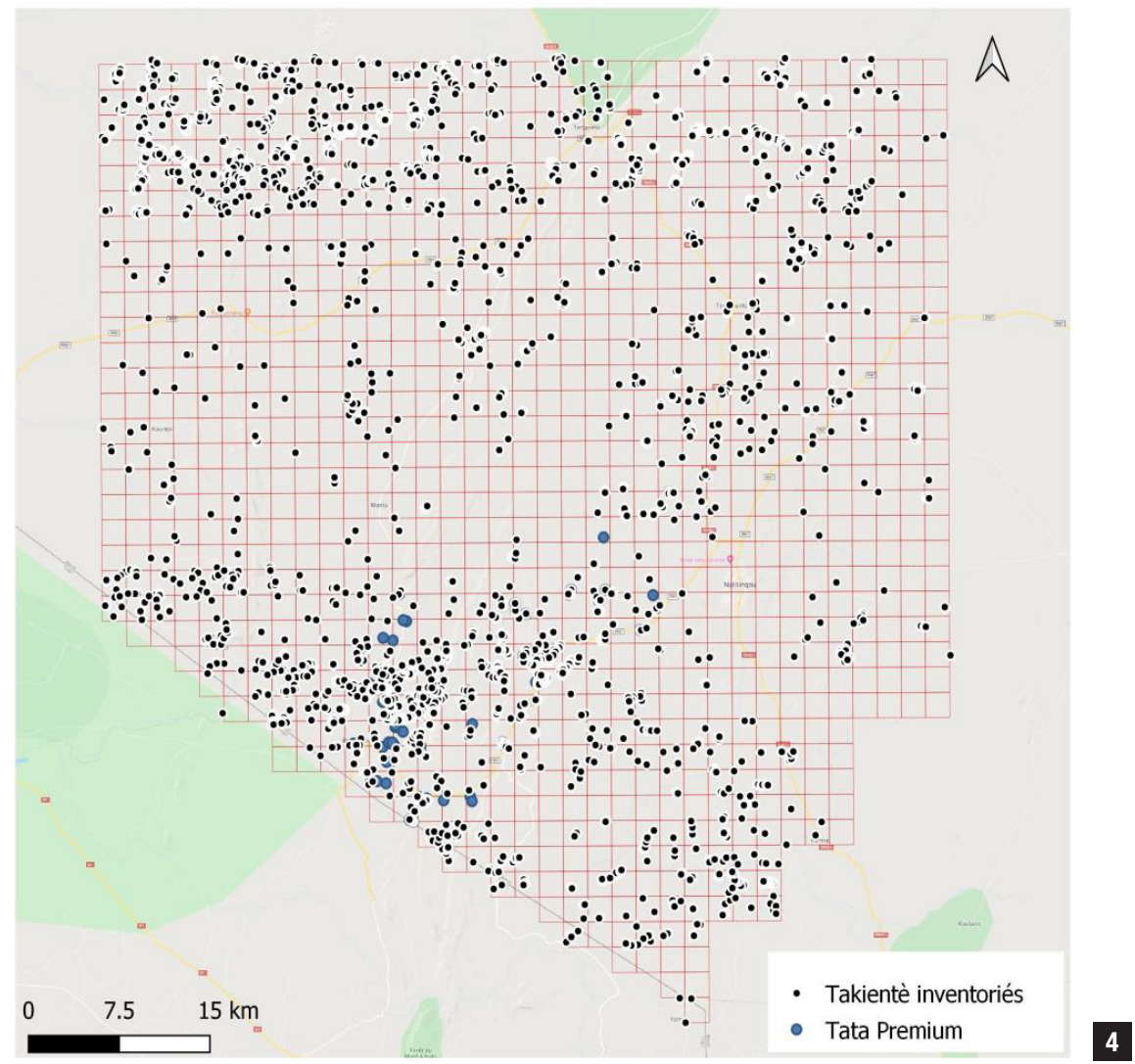

provient l'essentiel de la culture vivrière garante de l'autonomie alimentaire de la famille. Le village, quant à lui, qui se mesure en centaines de mètres, regroupe plusieurs domaines familiaux et des lieux collectifs (point d'eau, etc.) par l'intermédiaire de sentiers. Le territoire, enfin, mesurable en kilomètres, est constitué des villages et d'étendues arborées reliés entre eux par les réseaux routiers et hydrographiques.

Adoptant une approche générative, notre objectif est donc de décrire et d'expliquer les principes qui gouvernent l'intégration des entités architecturales dans des ensembles qui les dépassent. Traversant les quatre échelles, de la concession $^{9}$, du domaine, du village et du territoire, il s'agit de mettre à jour les logiques architecturales constitutives du "paysage otammari".

\section{Méthode}

Notre recherche se fonde sur des données récentes, auxquelles nos prédécesseurs n'ont pu avoir accès.

Nous nous appuyons d'abord sur un inventaire géolocalisé des takientè situés sur le territoire des communes de Boukoumbé, de Cobly, de Matéri, de Natitingou et de Tanguiéta, que nous avons réalisé nous-mêmes à partir d'images satellites datant de 2017, 2018 et 2019 et de photos aériennes au moyen du logiciel d'information géographique Qgis. II ressort de cet inventaire qu'il existe environ 4550 takientè (fig. 4), qui correspondent aux différents types de Tata Somba décrits par Puren ${ }^{10}$, ainsi qu'à de nouveaux modèles en expansion qui, hybrides et sans plateforme, sont inspirés de la structure initiale de la takienta

Au moyen du logiciel SPSS statistic 25, nous exploitons, ensuite, les informations contenues dans la base de données constituée en 2019 par l'Institut français de Cotonou dans le cadre de son projet de valorisation touristique "Route des Tata". Cette base de données contient en effet une grande variété d'informations géographiques, sociologiques et architecturales relatives à environ 1500 takientè, situées entre les communes de Boukoumbé et Natitingou, zone d'implantation initiale des Bètammaribè ${ }^{12}$.

Nous nous basons, en outre, sur les orthophotos de l'Atacora réalisés dans le cadre du projet HTC-Atacora ${ }^{13}$ (fig. 5). Obtenus au départ de photographies aériennes captées par des drones, ces orzaine de villages implantés dans l'Atacora.

Nous tirons parti, enfin, des cartes au 1/50 000 réalisées par l'Institut géographique national du Bénin, dont les données numériques sont accessibles depuis 2019 sur le Géoportail de I'IGNBénin, et exploitables par des logiciels d'information géographique tels que QGIS ou ArcGIS. assimilée au Kasséna ${ }^{11}$. thophotos présentent avec une grande précision la situation de fait d'une quin-
4 Inventaire géolocbalisé des tata somba (image : Fabrice Noukpakou)

9 - Espace à usage d'habitation regroupant dans une enceinte ou dans une cour des éléments aux fonctions diversifiées (habitation, réunion, etc.)

10 - PUREN, Odile (2010), op. cit.

11 - Architecture traditionnelle Gurunsi (Burkina Faso), peuple dont seraient en partie issus les Bètammaribè.

KAUCLEY, N'koué Benjamin, Contribution à l' histoire précoloniale des Bebelibe du territoire de Kobli (nordouest du Bénin) des origines à 1897 , Cotonou, Université d'AbomeyCalavi, 2016.

12 - N'DAH, Didier (2009), op. cit.,

13- Financé par l'Agence wallonne de l'air et du climat (AwAC), le projet HTC-ATACORA, porté par I'UCLouvain en partenariat avec Eco-Bénin, l'École du patrimoine africain et Yves Baudot, a pour objectif de préserver les habitats en terre crue et de promouvoir la construction d'habitats en terre crue résistants aux changements climatiques et peu consommateurs de bois de construction et de bois de chauffe. 
recherche

Ses données récentes que nous exploitons pour étudier le paysage otammari devraient nous amener à nuancer, voir à réfuter, quelques-unes des affirmations les plus fréquentes dans la littérature spécialisée, à savoir : que la takienta "peut-être envisagé seule"14; que "l'écartement entre les habitations est déterminé par la distance qu'une flèche pourrait parcourir" 15; que l'habitat somba "est un habitat dispersé"16.

\section{Conclusion}

L'habitat otammari ne se limite pas à la takienta. II englobe bien d'autres éléments, tout aussi importants et déterminants dans la vie quotidienne des Bètammaribè. Des éléments qui, de proche en proche, s'associent pour former une totalité que nous appelons, par commodité, "paysage". L'analyse générative de l'habitat otammari au départ de données qui couvrent ses différentes échelles nous permettra d'en décrire plus finement et d'en expliquer plus profondément les logiques d'implantation et d'articulation.
5 Village de Koussantikou (orthophotos: Yves Baudot, Fabrice Noukpakou)

14- MERCIER, Paul (1954), op.cit.

15- JOFFROY, Thierry et Nayondjoua DJANGUENANE, "Koutammakou"le pays des Batammariba "ceux quifaçonnent la terre", [Grenoble], CRATerre Editions, 2005

16 - PADENOU, Guy-Herman et Monique PASTOR-BARRUÉ (2006), op.cit.

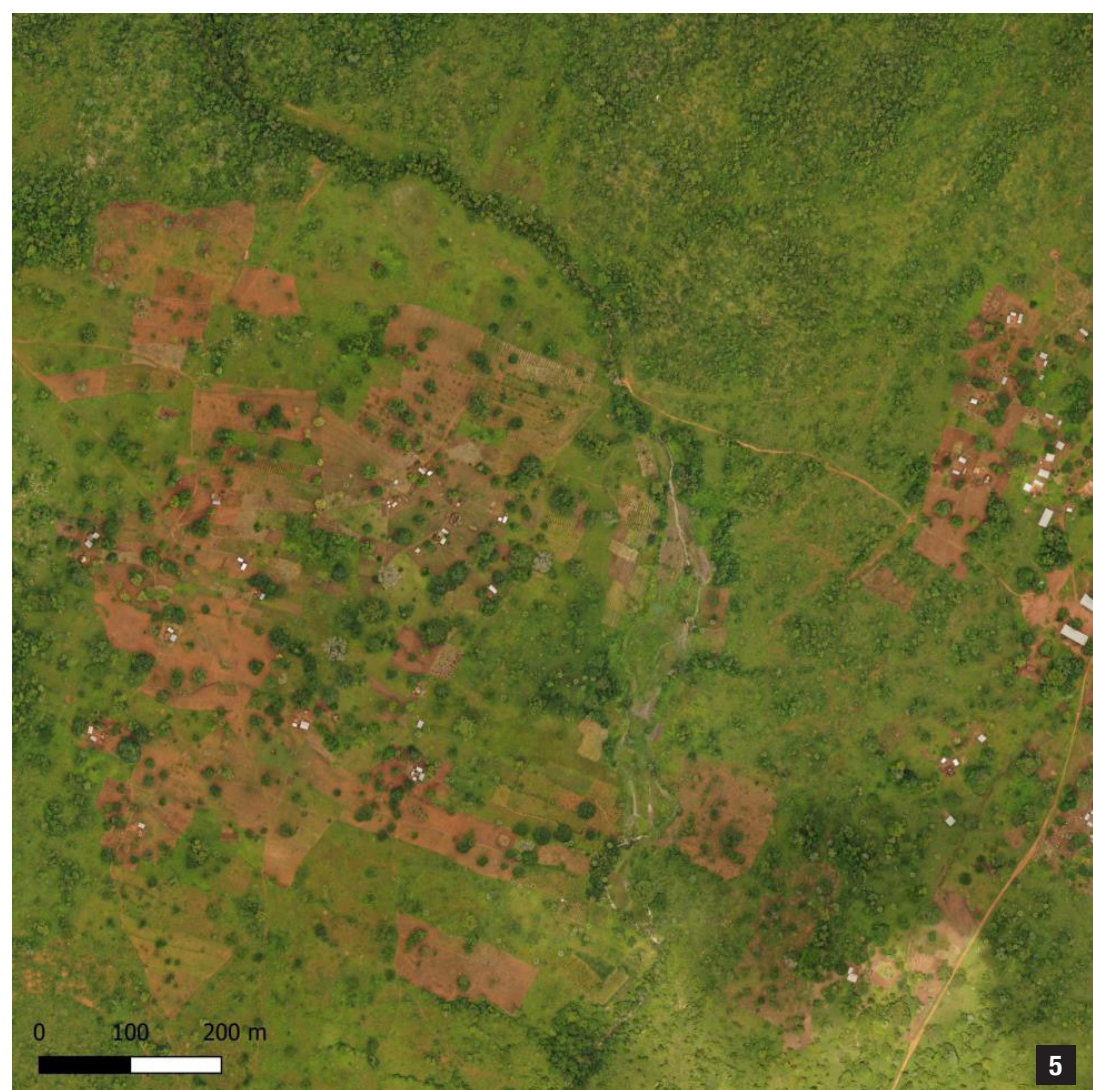

